Pacific Journal of Mathematics

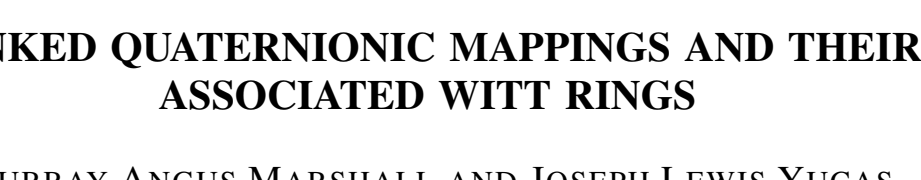




\title{
LINKED QUATERNIONIC MAPPINGS AND THEIR ASSOCIATED WITT RINGS
}

\author{
MURRay Marshall aNd Joseph YuCaS
}

A quaternionic mapping is a symmetric bilinear mapping $q: G \times G \rightarrow B$, where $G, B$ are Abelian groups, $G$ has exponent 2 and contains a distinguished element -1 such that $q(a, a)=$ $q(a,-1) \quad \forall a \in G$. Such a mapping is said to be linked if $q(a, b)=q(c, d)$ implies the existence of $x \in G$ such that $q(a, b)=q(a, x)$ and $q(c, d)=q(c, x)$. The Witt ring $W(q)$ of such a mapping $q$ can be defined to be the integral group ring $Z[G]$ factored by the ideal generated by $1+(-1)$ and the elements $(a+b)-(c+d)$ such that $a b=c d$ and $q(a, b)=$ $q(c, d)$. If $q$ is the quaternionic mapping associated to a field or semi-local ring $A$ with $2 \in A^{*}$, then $q$ is linked, and $W(q)$ is the Witt ring of free bilinear spaces over $A$. This paper gives a ring-theoretic description of the class of rings $W(q), q$ linked. In particular, all such rings are shown to be strongly representational in the terminology of Kleinstein and Rosenberg.

1. Introduction. Throughout this section, $F$ will denote a field or semi-local ring with $2 \in F^{*}$ such that all residue class fields contain more than 3 elements. Let $B_{F}$ denote the Brauer group of $F, G_{F}=F^{\cdot} / F^{\cdot 2}$, and let $q_{F}: G_{F} \times G_{F} \rightarrow B_{F}$ denote the quaternion algebra mapping. Then $q_{F}$ satisfies

(A) $q_{F}$ is symmetric and bilinear, i.e.,

$$
\forall a, b, c \in G_{F}, q_{F}(a, b)=q_{F}(b, a)
$$

and

$$
q_{F}(a, b c)=q_{F}(a, b) q_{F}(a, c) .
$$

(B) $\forall a \in G_{F}, q_{F}(a, a)=q_{F}(a,-1)$.

In the case $F$ is a field (A) is $[8,2.11, \mathrm{p} .61]$ and $(B)$ is $[8,2.6, \mathrm{p}$. 58]. The corresponding results for semi-local rings may be found in $[2$, p. 22-29].

It is well known that isometry of (quadratic) forms over $F$ is describable in terms of $q_{F}$. For forms of dimension one and two we have $(a) \cong(b) \Leftrightarrow a=b$, and $(a, b) \cong(c, d) \Leftrightarrow a b=c d$ and $q_{F}(a, b)=$ $q_{F}(c, d)$. The proof of this statement given for fields in $[8,2.9, p$. 60] will work as well in the semi-local ring case. For higher dimensional forms $f \cong g \Leftrightarrow \exists$ a sequence of forms $f=f_{0}, f_{1}, \cdots, f_{k}=g$ such that for each $i=1, \cdots, k, f_{i}$ is obtained from $f_{i-1}$ by replacing two diagonal entries $a, b$ by $c, d$ with $(a, b) \cong(c, d)$. For the 
proof of this last assertion, see [11, Satz 7] in case $F$ is a field, and [7, Lemma 1.14] in case $F$ is a semi-local ring.

In turn, this gives a description of the Witt ring $W_{F}$ of quadratic forms over $F$ in terms of $q_{F}$ : $W_{F}$ is the integral group ring $Z\left[G_{F}\right]$ factored by the ideal generated by $1+(-1)$ and the elements $(a+b)-(c+d)$ such that $a b=c d$ and $q_{F}(a, b)=q_{F}(c, d)$.

More generally, consider an abstract mapping $q: G \times G \rightarrow B$ where $G$ and $B$ are Abelian groups and $G$ has exponent 2 (i.e., $a^{2}=1 \forall a \in G$ ). If such a mapping satisfies properties (A) and (B) above for some distinguished element $-1 \in G$, we will say $q$ is a quaternionic mapping. If this is the case, we can certainly define isometry of (abstract) forms by the above formulas (see [4]), and construct an associated (abstract) Witt ring $W(q)$. Certainly some of the classical quadratic form theory will carry over to this abstract situation.

The goal of this paper is to develop a much more refined theory. The key observation is that $q_{F}$ has an additional important property.

$$
q_{F}(a, b)=q_{F}(c, d) \Rightarrow \exists x \in G_{F} \text { such that } q_{F}(a, b)=q_{F}(a, x)
$$

and $q_{F}(c, d)=q_{F}(c, x)$. In case $F$ is a field, this is an exercise in Lam's book $[8$, p. 69,12$]$. Here is a sketch of the proof in the semi-local case: First note that

$$
q_{F}(a, b)=q_{F}(c, d) \Leftrightarrow(1,-a) \otimes(1,-b) \cong(1,-c) \otimes(1,-d),
$$

using $[2,1.19$, p. 29]. Expanding and using Witt cancellation, this, in turn, is equivalent to $(-b, a b) \oplus(d,-c d) \cong(a,-c) \oplus(1,-1)$. Thus, by transversality $[3,2.7(\mathrm{c})], \exists x \in G_{F}$ such that $(-b, a b) \cong$ $(-x, a x)$ and $(d,-c d) \cong(x,-c x)$. It follows easily from this (for example, use (1) again), that $q_{F}(a, b)=q_{F}(a, x)$ and $q_{F}(c, d)=$ $q_{F}(c, x)$.

A quaternionic mapping $q: G \times G \rightarrow B$ is said to be linked if it satisfies (L). In this paper, we examine the form theory associated to a linked quaternionic mapping and develop properties of the associated Witt ring $W(q)$. In Theorem 2.6 the following cancellation property for forms is shown to hold:

$$
f \cong f^{\prime} \text { and } f \oplus g \cong f^{\prime} \oplus g^{\prime} \Rightarrow g \cong g^{\prime} .
$$

It follows from this that each form has a well-defined anisotropic part and Witt index, and that $W(q)$, as a set, can be described as the equivalence classes of forms with respect to Witt equivalence, exactly as in [11]. In Theorem 2.7, the following representation property for forms is proved: 


$$
D(f \oplus g)=\cup\{D(a, b) \mid a \in D(f), b \in D(g)\} .
$$

(Here, $D(f)$ denotes the set of elements of $G$ represented by the form $f$.) This implies that $W(q)$ is representational in the terminology of [5]. The exact relationship between linked quaternionic mappings and representational Witt rings is presented in Theorem 3.8 following the introduction of the signed discriminant and the Witt invariant. In Theorem 3.11, it is proved that $W(q)$ is reduced (i.e., has nilradical equal to zero) if and only if $q$ satisfies

$$
\forall a \in G, q(a, a)=1 \Rightarrow a=1 .
$$

This special case is of interest since, as pointed out in [5], the reduced representational Witt rings are just the Witt rings of spaces of orderings as presented, for example, in [9].

2. The form theory. Throughout, assume that $q: G \times G \rightarrow B$ is a linked quaternionic mapping. Recall, from the introduction, this means $G, B$ are Abelian groups, $G$ has exponent 2 and a distinguished element -1 , and $q$ satisfies

(A) $q$ is symmetric and bilinear,

(B) $q(a, a)=q(a,-1) \forall a \in G$, and

(L) $q(a, b)=q(c, d) \Rightarrow \exists x \in G$ such that $q(a, b)=q(a, x)$ and $q(c, d)=q(c, x)$.

It is worth pointing out, to begin with, that $\forall a, b \in G, q(a, b)^{2}=$ $q\left(a, b^{2}\right)=q(a, 1)=1$. In particular, the subgroup of $B$ generated by the image of $q$ has exponent 2 . Also, note that $q(a,-a)=$ $q(a,-1) q(a, a)=q(a,-1)^{2}=1$.

By a form of dimension $n \geqq 1$ (over $G$ ) is meant an $n$-tuple $f=\left(a_{1}, \cdots, a_{n}\right)$ with $a_{1}, \cdots, a_{n} \in G$. The discriminant and Hasse invariant of such a form $f$ are defined by

$$
d(f)=\prod_{i} a_{i} \text {, and } s(f)=\prod_{i>j} q\left(a_{i}, a_{j}\right) .
$$

The sum of $f$ and $g$, with $f$ as above and $g=\left(b_{1}, \cdots, b_{m}\right)$, is defined by $f \oplus g=\left(a_{1}, \cdots, a_{n}, b_{1}, \cdots, b_{m}\right)$. Isometry of one and two dimensional forms is defined by

(3) $(a) \cong(b) \Leftrightarrow a=b$, and

(4) $(a, b) \cong(c, d) \Leftrightarrow a b=c d$ and $q(a, b)=q(c, d)$.

For forms of dimension $n \geqq 3$, isometry is defined inductively by

$$
\left(a_{1}, \cdots, a_{n}\right) \cong\left(b_{1}, \cdots, b_{n}\right) \Leftrightarrow \exists a, b, c_{3}, \cdots, c_{n} \in G
$$

such that $\left(a_{2}, \cdots, a_{n}\right) \cong\left(a, c_{3}, \cdots, c_{n}\right),\left(b_{2}, \cdots, b_{n}\right) \cong\left(b, c_{3}, \cdots, c_{n}\right)$ and $\left(a_{1}, a\right) \cong\left(b_{1}, b\right)$. It will follow from 2.4 that this definition coincides with the one given in the introduction. 
THEOREM 2.1. If $b_{1}, \cdots, b_{n}$ is a permutation of $a_{1}, \cdots, a_{n}$, then $\left(a_{1}, \cdots, a_{n}\right) \cong\left(b_{1}, \cdots, b_{n}\right)$.

Proof. We may assume $n \geqq 3$. If $b_{1}=a_{i}, i \geqq 2$, take $a=a_{i}$, $b=a_{1}$, and take $c_{3}, \cdots, c_{n}$ to be the elements left after $a_{1}, a_{i}$ are deleted from $a_{1}, \cdots, a_{n}$. Note that $a, c_{3}, \cdots, c_{n}$ is a permutation of $a_{2}, \cdots, a_{n} ; b, c_{3}, \cdots, c_{n}$ is a permutation of $b_{2}, \cdots, b_{n}$; and $b_{1}, b$ is a permutation of $a_{1}, a$, so the result is true by induction. On the other hand, if $b_{1}=a_{1}$, take $a=b=a_{2}$, and $c_{i}=a_{i}, i \geqq 3$.

THEOREM 2.2. If $f \cong g$ then $\operatorname{dim}(f)=\operatorname{dim}(g), d(f)=d(g)$, and $s(f)=s(g)$. The converse holds for forms of dimension $n \leqq 3$.

Proof. It is clear that the theorem and its converse hold for 1 and 2 dimensional forms, by (3) and (4). (Note: if $f$ is 1-dimensional, then $s(f)=1$, by definition.) Now let $f=\left(a_{1}, \cdots, a_{n}\right), g=$ $\left(b_{1}, \cdots, b_{n}\right), n \geqq 3$. First suppose $f \cong g$, and choose $a, b, c_{3}, \cdots, c_{n}$ as in (5). Then, by induction, $a_{2} \cdots a_{n}=a c_{3} \cdots c_{n}, b_{2} \cdots b_{n}=b c_{3} \cdots c_{n}$, and $a_{1} a=b_{1} b$, so $a_{1} a_{2} \cdots a_{n}=a_{1} a c_{3} \cdots c_{n}=b_{1} b c_{3} \cdots c_{n}=b_{1} b_{2} \cdots b_{n}$. Also, using

$$
s(f \oplus h)=s(f) \cdot s(h) \cdot q(d(f), d(h))
$$

(this is easily verified), we have, by induction,

$$
\begin{aligned}
s(f) & =s\left(a_{2}, \cdots, a_{n}\right) q\left(a_{1}, a_{2} \cdots a_{n}\right) \\
& =s\left(a, c_{3}, \cdots, c_{n}\right) q\left(a_{1}, a c_{3} \cdots c_{n}\right) \\
& =s\left(c_{3}, \cdots, c_{n}\right) q\left(a, c_{3} \cdots c_{n}\right) q\left(a_{1}, a c_{3} \cdots c_{n}\right) \\
& =s\left(c_{3}, \cdots, c_{n}\right) q\left(a a_{1}, c_{3} \cdots c_{n}\right) q\left(a_{1}, a\right) \\
& =s\left(c_{3}, \cdots, c_{n}\right) q\left(b b_{1}, c_{3} \cdots c_{n}\right) q\left(b_{1}, b\right)=\cdots=s(g) .
\end{aligned}
$$

Now suppose $n=3, d(f)=d(g)$, and $s(f)=s(g)$. Thus $a_{3}=a_{1} a_{2} x$, $b_{3}=b_{1} b_{2} x$ where $x$ denotes the common discriminant. Thus using properties (A) and (B) of $q$,

$$
\begin{aligned}
& s\left(a_{1}, a_{2}, a_{1} a_{2} x\right)=q\left(a_{2}, a_{1} a_{2} x\right) q\left(a_{1}, a_{2}\right) q\left(a_{1}, a_{1} a_{2} x\right) \\
& \quad=q\left(a_{2}, a_{1} a_{2} x\right) q\left(a_{1}, a_{1} x\right)=q\left(a_{2}, a_{1} a_{2} x\right) q\left(a_{2},-a_{2}\right) q\left(-a_{1} x, a_{1} x\right) q\left(a_{1}, a_{1} x\right) \\
& \quad=q\left(a_{2},-a_{1} x\right) q\left(-x, a_{1} x\right)=q\left(a_{2},-a_{1} x\right) q\left(-x,-a_{1} x\right) q(-x,-1) \\
& \quad=q\left(-a_{2} x,-a_{1} x\right) q(-x,-1) .
\end{aligned}
$$

Here, as always, $-a$ denotes the element $(-1)(a) \in G$. We record this result:

$$
s\left(a_{1}, a_{2}, a_{1} a_{2} x\right)=q\left(-a_{1} x,-a_{2} x\right) q(-x,-1) .
$$

If we do the same computation for $g$, we see that the equality of 
the Hasse invariants implies $q\left(-a_{1} x,-a_{2} x\right)=q\left(-b_{1} x,-b_{2} x\right)$. Thus, by (L), $\exists y \in G$ such that $q\left(-a_{1} x,-a_{2} x\right)=q\left(-a_{1} x, y\right)$ and $q\left(-b_{1} x,-b_{2} x\right)=$ $q\left(-b_{1} x, y\right)$. Take $c_{3}=-x y, a=-a_{1} y$, and $b=-b_{1} y$. Now it is just a matter of checking $\left(a_{2}, a_{1} a_{2} x\right) \cong\left(a, c_{3}\right),\left(b_{2}, b_{1} b_{2} x\right) \cong\left(b, c_{3}\right)$ and $\left(a_{1}, a\right) \cong$ $\left(b_{1}, b\right)$. Clearly, the discriminants are the same and

$$
\begin{aligned}
q\left(a_{2}, a_{1} a_{2} x\right) & =q\left(a_{2}, a_{1} a_{2} x\right) q\left(a_{2},-a_{2}\right)=q\left(a_{2},-a_{1} x\right) \\
& =q\left(-a_{2} x,-a_{1} x\right) q\left(-x,-a_{1} x\right)=q\left(-a_{1} x, y\right) q\left(-x,-a_{1} x\right) \\
& =q\left(-a_{1} x,-x y\right)=q\left(-a_{1} x,-x y\right) q(x y,-x y)=q\left(-a_{1} y,-x y\right) \\
& =q\left(a, c_{3}\right) .
\end{aligned}
$$

Similarly $q\left(b_{2}, b_{1} b_{2} x\right)=q\left(b, c_{3}\right)$. Finally, using $q\left(-a_{1} x, y\right)=q\left(-b_{1} x, y\right)$, we have

$$
\begin{aligned}
q\left(a_{1}, a\right) & =q\left(a_{1},-a_{1} y\right)=q\left(a_{1}, y\right)=q\left(-a_{1} x, y\right) q(-x, y) \\
& =q\left(-b_{1} x, y\right) q(-x, y)=q\left(b_{1}, y\right)=q\left(b_{1},-b_{1} y\right)=q\left(b_{1}, b\right) .
\end{aligned}
$$

THEOREM 2.3. Isometry is a transitive relation.

(Note. Since isometry is clearly reflexive and symmetric, this implies it is an equivalence relation.)

Proof. Suppose $f, g, h$ are $n$ dimensional forms with $f \cong g \cong h$. We show $f \cong h$ by induction on $n$. By 2.2 , we may assume $n \geqq 4$. Let the elements $a, b, c \in G$ and the $n-1$ dimensional forms $f^{\prime}, g^{\prime}, h^{\prime}$ be defined by $f=(a) \oplus f^{\prime}, g=(b) \oplus g^{\prime}, \quad h=(c) \oplus h^{\prime}$. Thus, by assumption, $\exists a^{\prime}, b^{\prime}, b^{\prime \prime}, c^{\prime} \in G$ and $n-2$ dimensional forms $i, j$ such that $f^{\prime} \cong\left(a^{\prime}\right) \oplus i, g^{\prime} \cong\left(b^{\prime}\right) \oplus i, g^{\prime} \cong\left(b^{\prime \prime}\right) \oplus j, h^{\prime} \cong\left(c^{\prime}\right) \oplus j, \quad\left(a, a^{\prime}\right) \cong$ $\left(b, b^{\prime}\right)$, and $\left(b, b^{\prime \prime}\right) \cong\left(c, c^{\prime}\right)$. Thus, by induction, $\left(b^{\prime}\right) \oplus i \cong\left(b^{\prime \prime}\right) \oplus j$, so $\exists b_{1}, b_{2} \in G$ and an $n-3$ dimensional form $k$ satisfying $i \cong\left(b_{1}\right) \oplus k$, $j \cong\left(b_{2}\right) \oplus k$, and $\left(b^{\prime}, b_{1}\right) \cong\left(b^{\prime \prime}, b_{2}\right)$. It follows that $\left(a, a^{\prime}, b_{1}\right) \cong\left(b, b^{\prime}, b_{1}\right) \cong$ $\left(b, b^{\prime \prime}, b_{2}\right) \cong\left(c, c^{\prime}, b_{2}\right)$, so, using transitivity in the case $n=3, \exists a_{1}, c_{1}, x \in$ $G$ such that $\left(a^{\prime}, b_{1}\right) \cong\left(a_{1}, x\right),\left(c^{\prime}, b_{2}\right) \cong\left(c_{1}, x\right)$, and $\left(a, a_{1}\right) \cong\left(c, c_{1}\right)$. Take $l=(x) \oplus k$. Then $f^{\prime} \cong\left(a^{\prime}\right) \oplus i \cong\left(a^{\prime}, b_{1}\right) \oplus k \cong\left(a_{1}, x\right) \oplus k=\left(a_{1}\right) \oplus l$, and $h^{\prime} \cong\left(c^{\prime}\right) \oplus j \cong\left(c^{\prime}, b_{2}\right) \oplus k \cong\left(c_{1}, x\right) \oplus k=\left(c_{1}\right) \oplus l$. Thus, by induction, $f^{\prime} \cong\left(a_{1}\right) \oplus l$ and $h^{\prime} \cong\left(c_{1}\right) \oplus l$. Since $\left(a, a_{1}\right) \cong\left(c, c_{1}\right)$, this completes the proof.

CoROLLARY 2.4. $f \cong g \Leftrightarrow$ there exists a sequence of forms $f=f_{0}$, $f_{1}, \cdots, f_{k}=g, k \geqq 0$, such that for each $i=1, \cdots, k, f_{i}$ is obtained from $f_{i-1}$ by replacing two entries $a, a^{\prime}$ by $b, b^{\prime}$ respectively, where $\left(a, a^{\prime}\right) \cong\left(b, b^{\prime}\right)$.

Proof. The implication $(\Rightarrow)$ is immediate, by induction on 
$n=\operatorname{dim}(f)$. To prove $(\leftrightharpoons)$, we may assume $n \geqq 3$, and, by 2.3 , that $k=1$. Thus, by $2.1, f \cong\left(a, a^{\prime}, c_{3}, \cdots, c_{n}\right)$ and $g \cong\left(b, b^{\prime}, c_{3}, \cdots, c_{n}\right)$. Now it is clear $\left(a, a^{\prime}, c_{3}, \cdots, c_{n}\right) \cong\left(b, b^{\prime}, c_{3}, \cdots, c_{n}\right)$. Thus, by $2.3, f \cong g$.

Lemma 2.5. For arbitrary forms $f, g, g^{\prime}, g \cong g^{\prime} \Leftrightarrow f \oplus g \cong f \oplus g^{\prime}$.

Proof. We may assume $f$ is 1-dimensional, say $f=\left(a_{1}\right)$.

$(\Rightarrow)$ : Define $a, c_{3}, \cdots, c_{n}$ by $g=\left(a, c_{3}, \cdots, c_{n}\right)$ and let $b=a$. Then $f \oplus g \cong f \oplus g^{\prime}$ by (5).

$(\Leftarrow)$ : $\operatorname{By~(5)~} \exists a, b, c_{3}, \cdots, c_{n}$ such that $g \cong\left(a, c_{3}, \cdots, c_{n}\right), g^{\prime} \cong$ $\left(b, c_{3}, \cdots, c_{n}\right)$ and $\left(a_{1}, a\right) \cong\left(a_{1}, b\right)$. Comparing discriminants, this yields $a=b$, so $g \cong\left(a, c_{3}, \cdots, c_{n}\right) \cong g^{\prime}$. Thus $g \cong g^{\prime}$ by 2.3 .

THEOREM 2.6. Suppose $f, f^{\prime}, g, g^{\prime}$ are forms satisfying $f \cong f^{\prime}$. Then $g \cong g^{\prime} \Leftrightarrow f \oplus g \cong f^{\prime} \oplus g^{\prime}$.

Proof. Since $f \cong f^{\prime}$, it follows from 2.1 and 2.5 that $f \oplus g \cong$ $f^{\prime} \oplus g$. Thus, $f \oplus g \cong f^{\prime} \oplus g^{\prime} \Leftrightarrow f^{\prime} \oplus g \cong f^{\prime} \oplus g^{\prime} \Leftrightarrow g \cong g^{\prime}$ by 2.3 and 2.5 .

For $f=\left(a_{1}, \cdots, a_{n}\right), \quad g=\left(b_{1}, \cdots, b_{m}\right)$ and $a \in G$ let us define $a f:=\left(a a_{1}, \cdots, a a_{n}\right)$, and $f \otimes g:=\left(a_{1} b_{1}, \cdots, a_{1} b_{m}, \cdots, a_{n} b_{1}, \cdots, a_{n} b_{m}\right)$. (Thus $a f=(a) \otimes f$.)

THEOREM 2.7. (i ) If $f \cong f^{\prime}$, then $a f \cong a f^{\prime}$.

(ii) If $f \cong f^{\prime}$ and $g \cong g^{\prime}$, then $f \otimes g \cong f^{\prime} \otimes g^{\prime}$.

Proof. Let $f=\left(a_{1}, \cdots, a_{n}\right)$. (i) is clear if $n=1$. Suppose $n=2$, and that $f^{\prime}=\left(a_{1}^{\prime}, a_{2}^{\prime}\right)$. Then $a_{1} a_{2}=a_{1}^{\prime} a_{2}^{\prime}$ and $q\left(a_{1}, a_{2}\right)=q\left(a_{1}^{\prime}, a_{2}^{\prime}\right)$. It follows that $a f$ and $a f^{\prime}$ have the same discriminant and $q\left(a a_{1}, a a_{2}\right)=$ $q(a, a) q\left(a, a_{1} a_{2}\right) q\left(a_{1}, a_{2}\right)=q(a, a) q\left(a, a_{1}^{\prime} a_{2}^{\prime}\right) q\left(a_{1}^{\prime}, a_{2}^{\prime}\right)=q\left(a a_{1}^{\prime}, a a_{2}^{\prime}\right)$. Thus $a f \cong$ $a f^{\prime}$. The result for $n \geqq 3$ follows by a simple inductive argument.

To prove (ii), note $f \otimes g \cong a_{1} g \oplus \cdots \oplus a_{n} g \cong a_{1} g^{\prime} \oplus \cdots \oplus a_{n} g^{\prime} \cong$ $f \otimes g^{\prime}$, using part (i) and 2.6. Similarly $f \otimes g^{\prime} \cong f^{\prime} \otimes g^{\prime}$, so $f \otimes g \cong$ $f^{\prime} \otimes g^{\prime}$.

We say a form $f$ of dimension $n$ represents $x \in G$ if $\exists x_{2}, \cdots$, $x_{n} \in G$ such that $f \cong\left(x, x_{2}, \cdots, x_{n}\right)$. Let us denote by $D(f)$ the set of elements $x \in G$ represented by $f$ in this sense.

THEOREM 2.8. If $f$ and $g$ are arbitrary forms, then

$$
D(f \oplus g)=\cup\{D(x, y) \mid x \in D(f), y \in D(g)\} .
$$

Proof. To prove the nontrivial inclusion let $f=\left(a_{1}, \cdots, a_{k}\right)$, 
$g=\left(a_{k+1}, \cdots, a_{n}\right)$, and suppose $f \oplus g \cong\left(b_{1}, \cdots, b_{n}\right)$. Choose $a, b, c_{3}, \cdots, c_{n}$ as in (5). Thus $b_{1} \in D\left(a_{1}, a\right)$. This completes the proof if $k=1$ (take $x=a_{1}, y=a$ ). If $k \geqq 2$, then, by induction on $k, \exists x^{\prime} \in$ $D\left(a_{2}, \cdots, a_{k}\right), y \in D(g)$ such that $a \in D\left(x^{\prime}, y\right)$. Thus, $b_{1} \in D\left(a_{1}, a\right) \leqq$ $D\left(a_{1}, x^{\prime}, y\right)=D\left(y, a_{1}, x^{\prime}\right)$, so by the case $k=1, \exists x \in D\left(a_{1}, x^{\prime}\right)$ such that $b_{1} \in D(y, x)=D(x, y)$. Since $D\left(a_{1}, x^{\prime}\right) \subseteq D(f)$, this completes the proof.

Note that $(a,-a) \cong(1,-1) \forall a \in G$ by (4), since $q(a,-a)=1=$ $q(1,-1)$. Any form $(a,-a), a \in G$ will be called a hyperbolic form. A form $f$ will be called isotropic if $\exists$ a form $g$ such that $f \cong$ $(1,-1) \oplus g$. Otherwise $f$ will be called anisotropic. The following version of 2.8 is useful.

CoRollary 2.9. Let $f, g$ be forms, and suppose $f \oplus g$ is isotropic. Then $\exists x \in D(f)$ such that $-x \in D(g)$.

Proof. (Compare to [5, 2.4] and [9, 2.2].) Let $a, f^{\prime}$, and $h$ be such that $f=(a) \oplus f^{\prime}$ and $f \oplus g \cong(1,-1) \oplus h \cong(a,-a) \oplus h$. Thus $f^{\prime} \oplus g \cong(-a) \oplus h$ by 2.6. Suppose $\operatorname{dim}\left(f^{\prime}\right) \geqq 1$. Then, by 2.8, $\exists b \in D(g), \quad c \in D\left(f^{\prime}\right), \quad d \in G$ such that $(b, c) \cong(-a, d)$. Adding $(a,-b)$ to both sides and cancelling using 2.6 , this yields $(a, c) \cong$ $(-b, d)$. Thus, $-b \in D(a, c) \subseteq D(f)$, i.e., $x=-b$ satisfies the required conditions. If, on the other hand, $\operatorname{dim}\left(f^{\prime}\right)=0$, then $x=a$ works.

3. The Witt ring. We can now define the Witt ring associated to the linked quaternionic mapping $q$ exactly as in [11]. First note that every form $f$ over $G$ decomposes as

$$
f \cong f_{a} \oplus k \times(1,-1)
$$

with $f_{a}$ an anisotropic (possibly zero dimensional) form, and $k \geqq 0$. Here, $k \times g$ denotes $g \oplus \cdots \oplus g$ ( $k$ times) or the zero dimensional form if $k=0$. Using the cancellation property $2.6, k$ is uniquely determined by $f$, and $f_{a}$ is determined, up to isometry, by $f$. Let us refer to $f_{a}$ as the anisotropic part of $f$, to $k$ as the Witt index of $f$, and to (8) as the Witt decomposition of $f$.

Two forms $f, g$ (not necessarily of the same dimension) are said to be Witt equivalent, denoted $f \sim g$, if their anisotropic parts are isometric. It is clear that

$$
f \sim g \Rightarrow \operatorname{dim}(f) \equiv \operatorname{dim}(g)(\bmod 2)
$$

and

$$
f \cong g \Leftrightarrow f \sim g \text { and } \operatorname{dim}(f)=\operatorname{dim}(g)
$$


Let us denote by $W$ the set of equivalence classes of forms with respect to Witt equivalence. It is easily verified, using 2.6 and 2.7, that $\oplus$ and $\otimes$ induce binary operations on $W$, and by the same elementary arguments as in [11], $W$ becomes a commutative ring with unity. We will refer to the ring $W$ so constructed as the Witt ring associated to $q$, and will denote this by writing $W=W(q)$.

We remark in passing that we have the following description of $W(q)$.

THEOREM 3.1. $W(q)$ is isomorphic to the integral group ring $Z[G]$ factored by the ideal generated by $1+(-1)$ and the elements $(a+b)-(c+d)$ where $(a, b) \cong(c, d)$.

Proof. On the basis of 2.4 the proof is the same as in the classical case, ef. [8, Exc. 1, p. 49].

Denote by $I(q)$ the ideal of even dimensional forms in $W(q)$. Clearly $W(q) / I(q) \cong Z / 2 Z$. Since $(a, b) \sim(1, a)-(1,-b), I(q)$ is generated additively by the 1-fold Pfister forms $(1,-a), a \in G$. Thus $I^{k}(q)$ is generated additively by the $k$-fold Pfister forms $\left(1,-a_{1}\right) \otimes$ $\left(1,-a_{2}\right) \otimes \cdots \otimes\left(1,-a_{k}\right), a_{1}, \cdots, a_{k} \in G$.

We now modify the discriminant and Hasse invariant in a standard way (eg. see [8, p. 123]) to obtain invariants with respect to Witt equivalence. Namely, we define the signed discriminant and the Witt invariant by

$$
d_{ \pm}(f)=(-1)^{\alpha} d(f), \text { where } \alpha=n(n-1) / 2, n=\operatorname{dim}(f),
$$

and

$$
w(f)=s(f) q(-1, d(f))^{\varepsilon} q(-1,-1)^{\eta},
$$

where $\varepsilon=(n-1)(n-2) / 2, \eta=(n+1)(n)(n-1)(n-2) / 24$, and $n=$ $\operatorname{dim}(f)$.

THEOREM 3.2. (i ) $d_{ \pm}: W(q) \rightarrow G$ is well-defined.

(ii) The restriction of $d_{ \pm}$to the additive group $I(q)$ is a group homomorphism.

(iii) $I(q) / I^{2}(q) \cong G$.

Proof. Suppose $f=g$ in $W(q)$. We may assume $f \cong g \oplus k \times(1,-1)$ for some $k \in Z$. By 2.2, $d(f)=d(g)$ if $k \equiv 0(\bmod 4)$ and $d(f)=-d(g)$ if $k \equiv 2(\bmod 4)$. Consequently, $d_{ \pm}(f)=d_{ \pm}(g)$ and (i) is proved. Suppose $f_{1}, f_{2} \in I(q)$ and $\operatorname{dim} f_{1}=m_{1}, \operatorname{dim} f_{2}=m_{2}$. Then 


$$
\begin{aligned}
d_{ \pm}\left(f_{1} \oplus f_{2}\right) & =(-1)^{\left(m_{1}+m_{2}\right)\left(m_{1}+m_{2}-1\right) / 2} d\left(f_{1} \oplus f_{2}\right) \\
& =(-1)^{m_{1}\left(m_{1}-1\right) / 2}(-1)^{m_{2}\left(m_{2}-1\right) / 2}(-1)^{m_{1} m_{2}} d\left(f_{1}\right) d\left(f_{2}\right)=d_{ \pm}\left(f_{1}\right) d_{ \pm}\left(f_{2}\right)
\end{aligned}
$$

and (ii) is proved. Since $d_{ \pm}((1,-a) \otimes(1,-b))=d_{ \pm}(1,-a,-b, a b)=1$, the kernel of $d_{ \pm}: I(q) \rightarrow G$ contains $I^{2}(q)$. Since $(1,-a) \oplus(1,-b) \sim$ $(1,-a b) \oplus(1,-a) \otimes(1,-b)$, every element $f \in I(q)$ has the form $f=(1,-a)$ modulo $I^{2}(q)$. Hence $d_{ \pm}(f)=1 \Leftrightarrow d_{ \pm}(1,-a)=1 \Leftrightarrow a=$ $1 \Rightarrow f \in I^{2}(q)$. Thus the kernel is exactly $I^{2}(q)$. This proves (iii).

THEOREM 3.3. (i) If $f$ is an arbitrary form and $g$ is a form satisfying $d_{ \pm}(g)=1, \operatorname{dim}(g) \equiv 0(\bmod 2)$, then $w(f \oplus g)=w(f) w(g)$.

(ii) $w: W(q) \rightarrow B$ is well-defined.

(iii) $\quad w: I^{2}(q) \rightarrow B$ is a group homomorphism with $I^{3}(q) \subseteq \operatorname{ker}(w)$.

Proof. (i ) Note that $\operatorname{dim}(f \oplus g)=\operatorname{dim}(f)+\operatorname{dim}(g), d(f \oplus g)=$ $d(f) d(g)$ and $s(f \oplus g)=s(f) s(g) q(d(f), d(g))$ by (6). By hypothesis, $\operatorname{dim}(g)=2 k$, and $d_{ \pm}(g)=1$, so $d(g)$ is either 1 or -1 depending on whether $k$ is even or odd. The conclusion of (i) now follows from a lengthy (but elementary) computation.

(ii) Taking $g=(1,-1)$ in (i), we have $w(f \oplus(1,-1))=$ $w(f) w(1,-1)=w(f)$. It follows from this and 2.2, that $f \sim h \Rightarrow$ $w(f)=w(h)$.

(iii) By 3.2, $I^{2}(q)$ consists of those elements of $W(q)$ represented by forms $f$ satisfying $\operatorname{dim}(f) \equiv 0(\bmod 2)$ and $d_{ \pm}(f)=1$. Thus the fact that $w: I^{2}(q) \rightarrow B$ is a group homomorphism is a special case of (i). Finally observe that

$$
\begin{aligned}
& s(a(1,-b) \otimes(1,-c))=s(a,-a b,-a c, a b c) \\
& \quad=q(a, a) q(-a b,-b) q(-a c, a b c)=q(a, a) q(-a,-b) q(-a c, b) \\
& \quad=q(a, a) q(-a,-1) q(c, b)=q(-1,-1) q(b, c) .
\end{aligned}
$$

It follows that

$$
w(a(1,-b) \otimes(1,-c))=q(b, c) \quad \forall a, b, c \in G .
$$

Thus,

$$
\begin{aligned}
& w((1,-a) \otimes(1,-b) \otimes(1,-c))=w((1,-b) \otimes(1,-c) \\
& \oplus-a(1,-b) \otimes(1,-c))=q(b, c) q(b, c)=1 \quad \forall a, b, c \in G,
\end{aligned}
$$

so $I^{3}(q) \subseteq \operatorname{ker}(w)$.

CoROLlaRY 3.4. Let $a, b, c, d \in G$. Then the following are equivalent.

(i ) $q(a, b)=q(c, d)$, 
(ii) $\quad(1,-a) \otimes(1,-b) \cong(1,-c) \otimes(1,-d)$,
(iii) $(1,-a) \otimes(1,-b) \equiv(1,-c) \otimes(1,-d)\left(\bmod I^{3}(q)\right)$.

Proof. By (7), $s(-a,-b, a b)=q(a, b) q(-1,-1)$, so (i ) $\Rightarrow$ $(-a,-b, a b) \cong(-c,-d, c d)$ by 2.2 . This, in turn, clearly implies (ii). The implication (ii) $\Rightarrow$ (iii) is clear. Finally, if one applies $w$ to each member of (iii) and uses (13) and 3.3 (iii), one obtains (i).

Suppose $q_{i}: G_{i} \times G_{i} \rightarrow B_{i}$ is a linked quaternionic mapping, $i=1,2$. We will say $q_{1}$ and $q_{2}$ are equivalent, denoted $q_{1} \sim q_{2}$, if $\exists$ a group isomorphism $\alpha: G_{1} \cong G_{2}$ such that $\alpha(-1)=-1$ and $q_{1}(a, b)=1 \Leftrightarrow$ $q_{2}(\alpha(a), \alpha(b))=1 \forall a, b \in G_{1}$. Note that $q_{1} \sim q_{2}$ implies

$$
\begin{aligned}
q_{1}(a, b) & =q_{1}(c, d) \Leftrightarrow q_{2}(\alpha(a), \alpha(b)) \\
& =q_{2}(\alpha(c), \alpha(d)) \quad \forall a, b, c, d \in G_{1} .
\end{aligned}
$$

This follows since $q(a, b)=q(c, d) \Leftrightarrow \exists x \in G$ such that $q(a, b x)=1$, $q(c, d x)=1$ and $q(a c, x)=1$, by the linkage condition.

Corollary 3.5. Define $q^{\prime}: G \times G \rightarrow I^{2}(q) / I^{3}(q)$ by $q^{\prime}(a, b)=(1$, $-a) \otimes(1,-b)+I^{3}(q)$. Then $q^{\prime}$ is a linked quaternionic mapping and $q \sim q^{\prime}$.

Proof. This is clear, using 3.2 and 3.4 .

CoROLlaRY 3.6. Let $q_{i}: G_{i} \times G_{i} \rightarrow B_{i}$ be a linked quaternionic mapping, $i=1,2$. Then $q_{1} \sim q_{2} \Leftrightarrow W\left(q_{1}\right) \cong W\left(q_{2}\right)$.

Proof. $\quad \Leftrightarrow)$ : In view of the definition of $W\left(q_{i}\right)$, it is enough to verify $(a, b) \cong(c, d) \Leftrightarrow(\alpha(a), \alpha(b)) \cong(\alpha(c), \alpha(d)) \quad \forall a, b, c, d \in G$. This follows from (14) and the fact that $\alpha$ is a group isomorphism. $(\Longleftarrow)$ : In view of 3.5 , it is enough to show $q_{1}^{\prime} \sim q_{2}^{\prime}$. Now it is clear (since $I\left(q_{i}\right)$ can be characterized as the unique ideal of index 2 in $\left.W\left(q_{i}\right)\right)$ that the given isomorphism $\varphi: W\left(q_{1}\right) \rightarrow W\left(q_{2}\right)$ carries $I^{k}\left(q_{1}\right)$ onto $I^{k}\left(q_{2}\right) \quad \forall k \geqq 1$, and hence induces isomorphisms $I^{2}\left(q_{1}\right) / I^{3}\left(q_{1}\right) \cong$ $I^{2}\left(q_{2}\right) / I^{3}\left(q_{2}\right)$ and $G_{1} \cong I\left(q_{1}\right) / I^{2}\left(q_{1}\right) \cong I\left(q_{2}\right) / I^{2}\left(q_{2}\right) \cong G_{2}$ (using 3.2). Moreover, we claim that the following diagram

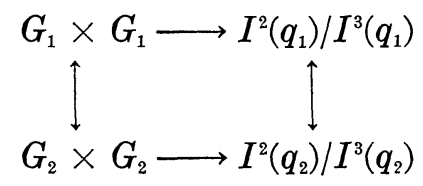

commutes. First recall as in 3.2 (iii) that for every $x \in G_{1}, \phi(1, x)$ can be written in the form $(1, y) \oplus f$ for some $y \in G_{2}$ and $f \in I^{2}\left(q_{2}\right)$. 
Consequently, for $a_{1}, b_{1} \in G_{1}$ we have

$$
\begin{aligned}
& \downarrow_{\left(a_{2}, b_{2}\right)}^{\left(a_{1}, b_{1}\right)} \longrightarrow\left(1,-a_{1},-b_{1}, a_{1} b_{1}\right)+I^{3}\left(q_{1}\right) \\
& \longrightarrow\left(1,-a_{2},-b_{2}, a_{2} b_{2}\right)+I^{3}\left(q_{2}\right)
\end{aligned}
$$

where $\varphi\left(1,-a_{1}\right)=\left(1,-a_{2}\right) \oplus f_{1}$ and $\varphi\left(1,-b_{1}\right)=\left(1,-b_{2}\right) \oplus f_{2}$ with $f_{1}, f_{2} \in I^{2}\left(q_{2}\right)$. Now, by an elementary computation it follows that $\varphi\left(\left(1,-a_{1},-b_{1}, a_{1} b_{1}\right)+I^{3}\left(q_{1}\right)\right)=\left(1,-a_{2},-b_{2}, a_{2} b_{2}\right)+I^{3}\left(q_{2}\right)$ and the diagram commutes. Finally, since the isomorphisms $G_{i} \cong I\left(q_{i}\right) / I^{2}\left(q_{i}\right)$ carry -1 to 2 and $2 \in I\left(q_{1}\right)$ is mapped to $2 \in I\left(q_{2}\right)$, the isomorphism $G_{1} \cong G_{2}$ carries -1 to -1 . This proves $q_{1}^{\prime} \sim q_{2}^{\prime}$.

In case $q=q_{F}, F$ a field, the following Arason-Pfister property is known to hold $\forall k \geqq 2$.

$\operatorname{AP}(k)$ : If $f$ is a form satisfying $\operatorname{dim}(f)<2^{k}$ and $f \in I^{k}(q)$, then $f \sim 0$.

For the proof see [1]. It is open whether this is true for $F$ a semi-local ring. However we do have the following.

COROLLARY 3.7. For $q$ an arbitrary linked quaternionic mapping, $\mathrm{AP}(k)$ holds for $k=2$, and 3 .

Proof. Let $\operatorname{dim}(f)<2^{k}, f \in I^{k}(q)$. Suppose first that $k=2$, $f=(a, b)$. Applying $d_{ \pm}$this yields $-a b=1$, by 3.2, i.e., $b=-a$. Thus $f=(a,-a) \sim 0$. Now suppose $k=3$. Adding enough hyperbolic forms, we can assume $f=\left(a_{1}, a_{2}, \cdots, a_{8}\right)$. Scaling $f$ by $a_{1} a_{2} a_{3}$, if necessary, we can assume $a_{3}=a_{1} a_{2}$. By 3.2, $d_{ \pm}(f)=1$, i.e., $a_{6}=$ $-a_{4} a_{5}$. Thus $f=\left(a_{1}, a_{2}, a_{1} a_{2}, a_{4}, a_{5},-a_{4} a_{5}\right) \sim\left(1, a_{1}\right) \otimes\left(1, a_{2}\right)-\left(1,-a_{4}\right) \otimes$ $\left(1,-a_{5}\right) \in I^{3}(q)$, so $f \sim 0$ by 3.4 .

We now relate the theory just presented with the theoy of representational Witt rings developed in [5]. For the reader's convenience we first record some definitions. Let $G$ be a group of exponent 2. A ring $W=Z[G] / K$ is called an abstract Witt ring if the torsion subgroup of $W$ is 2-primary, [7, Def. 3.12]. Throughout this section we will assume without loss of generality that $G$ is a subgroup of the multiplicative group $W^{\circ}$, and that $-1 \in G$ (simply replace $G$ by the subgroup of $W^{\cdot}$ generated by its image and -1). For $r \in W, \operatorname{dim} r$ is the smallest number $n$ such that $r=\sum_{i=1}^{n} g_{i}$ in $W, g_{i} \in G$, and $D(r)=\{g \in G \mid r=g+p$ for some $p \in W$ with $\operatorname{dim} p<\operatorname{dim} r$ \}, [5, Def. 1.1 and Def. 1.2]. $W$ will be called representational if for $r_{1} \neq 0, r_{2} \neq 0$ in $W$ with $\operatorname{dim}\left(r_{1}+r_{2}\right)=$ $\operatorname{dim} r_{1}+\operatorname{dim} r_{2}$ and $g$ in $D\left(r_{1}+r_{2}\right)$, there exist $g_{j}$ in $D\left(r_{j}\right), j=1,2$ 
such that $g \in D\left(g_{1}+g_{2}\right)$, [5, Def 2.2]. $W$ is strongly representational if for $g_{1}, g_{2} \in G$, with $g_{1}+g_{2} \neq 0$ in $W$ and $g \in D\left(g_{1}+g_{2}\right)$ we have $g+g g_{1} g_{2}=g_{1}+g_{2}$, [5, Def 4.1].

It is convenient to associate to $W$ a theory of forms. Namely, for $a_{i}, b_{j} \in G$, one defines $\left(a_{1}, \cdots, a_{n}\right) \sim\left(b_{1}, \cdots, b_{m}\right)$ to mean $a_{1}+\cdots+$ $a_{n}=b_{1}+\cdots+b_{m}$ in $W$ and $\left(a_{1}, \cdots, a_{n}\right) \cong\left(b_{1}, \cdots, b_{m}\right)$ to mean $\left(a_{1}, \cdots, a_{n}\right) \sim\left(b_{1}, \cdots, b_{m}\right)$ and $n=m$. Isometry so defined clearly satisfies $2.1,2.3,2.6,2.7$. Notice that our definitions of dimension determinant, representation, isotropic and anisotropic also make sense for this definition of isometry. Now, $W$ is representational if and only if 2.8 holds for forms over $W$. This follows quite easily from [5, Prop. 2.29]. Since 2.9 follows from 2.8, 2.9 also holds if $W$ is representational. Now, suppose $W$ is representational and $\left(a_{1}, \cdots, a_{n}\right) \cong\left(b_{1}, \cdots, b_{n}\right)$. There exists $a \in D\left(a_{2}, \cdots, a_{n}\right)$ such that $\left(a_{1}, a\right) \cong\left(b_{1}, b\right)$ for some $b \in G$, by 2.8. Since $a \in D\left(a_{2}, \cdots, a_{n}\right)$ there exist $c_{3}, \cdots, c_{n} \in G$ such that $\left(a_{2}, \cdots, a_{n}\right) \cong\left(a, c_{3}, \cdots, c_{n}\right)$. Consequently,

$$
\begin{aligned}
\left(a_{1}, a, b_{2}, \cdots, b_{n}\right) & \cong\left(b_{1}, b, b_{2}, \cdots, b_{n}\right) \cong\left(b, a_{1}, \cdots, a_{n}\right) \\
& \cong\left(b_{1}, a_{1}, a, c_{3}, \cdots, c_{n}\right)_{2},
\end{aligned}
$$

so $\left(b \cdots, b_{n}\right) \cong\left(b_{1}, c_{3}, \cdots, c_{n}\right)$ and (5) holds. 2.4 and 3.1 hold also by the same arguments given earlier. Clearly $W$ is strongly representational if and only if $(a, b) \cong(c, d) \Rightarrow a b=c d$ and hence (by an easy application of 2.4) if and only if $f \cong q \Rightarrow d(f)=d(g)$. Consequently, 3.2 holds and hence AP (2) holds (by the proof of 3.7) for strongly representational Witt rings. This proves part of the following.

THEOREM 3.8. Let $W$ be an abstract Witt ring for $G$ (with $G$ normalized so that $\left.-1 \in G \subseteq W^{*}\right)$. Then

(i) $W$ is strongly representational for $G \Leftrightarrow W$ is representational and satisfies AP (2) for G.

(ii) There exists a linked quaternionic mapping $q: G \times G \rightarrow B$ such that $W=W(q) \Leftrightarrow W$ is representational and satisfies $\operatorname{AP}(k)$, $k=2,3$, for $G$.

Proof. (i ) We have just proved $(\Leftrightarrow)$. To prove $\Leftrightarrow$ suppose $a+b=c+d$ with $a, b, c, d \in G$. Then $a b-c d=a(a+b)-c(c+d)=$ $a(a+b)-c(a+b)=(a-c)(a+b) \in I^{2}$. By AP (2), this implies $a b-$ $c d=0$, i.e., $a b=c d$.

(ii) If $q$ is linked, then $W(q)$ is an abstract Witt ring for $G$ by 3.1 , it is representational by 2.8 , and satisfies $\operatorname{AP}(k), k=2,3$ by 3.7. This proves $(\Leftrightarrow)$. To prove $\Leftrightarrow$ ) define $q: G \times G \rightarrow I^{2} / I^{3}$ by $q(a, b)=(1-a)(1-b)+I^{3}$, where $I$ is the unique ideal of index 2 . 
Since $(1-b c) \equiv(1-b)+(1-c)\left(\bmod I^{2}\right)$ and $(1-a)^{2}=2(1-a), q$ is clearly a quaternionic mapping. Note $(1-a)(1-b) \equiv(1-c)(1-d)$ $\left(\bmod I^{3}\right) \Leftrightarrow-a-b+a b+c+d-c d \in I^{3} \Leftrightarrow-a-b+a b+c+d-$ $c d=0 \Leftrightarrow(1-a)(1-b)=(1-c)(1-d)$ by AP (3). Thus, if $q(a, b)=$ $q(c, d)$, then $(-b+a b)+(d-c d)=a-c$ so by $2.9, \mathrm{AP}(2)$ and part (i) $\exists x \in G$ such that $-b+a b=-x+a x$ and $d-c d=x-c x$. This implies $q(a, b)=q(a, x)$ and $q(c, d)=q(c, x)$ so $q$ is linked. It follows from 3.1 and the corresponding structure result for $W$ that $W=$ $W(q)$.

It is shown in $[7, \S 3]$ that some of the structure results in [10] concerning the nilradical and the reduced Witt ring hold for any abstract Witt ring. For easy reference, we now summarize some of these results. For $W$ an abstract Witt ring, denote by $W_{t}, X, I$, and $\mathrm{Nil}(W)$, the torsion subgroup, the set of signatures (i.e., ring homomorphisms $\sigma: W \rightarrow Z$ ), the unique ideal of index 2, and the nilradical, respectively, of $W$.

THEOREM 3.9. Let $W$ be an abstract Witt ring. Then

(i) $W_{t}$ is 2-primary,

(ii) $W_{t}=\{f \in W \mid \sigma(f)=0 \forall \sigma \in X\}$, and

(iii) $\operatorname{Nil}(W)=W_{t} \cap I$.

(More precisely, in (iii), since $W_{t} \leqq I$ if $X \neq \Phi$, whereas $I \leqq W_{t}=$ $W$, if $X=\Phi$, one has $\operatorname{Nil}(W)=W_{t}$, if $W_{t} \neq W$, and $\operatorname{Nil}(W)=I$, if $\left.W_{t}=W.\right)$

The following result is useful in verifying $\mathrm{AP}(k)$ in certain cases.

Lemma 3.10. Suppose $W$ is an abstract Witt ring for $G$ with $-1 \in G \cong W^{\cdot}$. If $I^{k}$ is torsion free, then $\mathrm{AP}(k)$ holds.

Proof. Suppose $f$ is a form over $G, f \in I^{k}, \operatorname{dim}(f)<2^{k}$. Let $\sigma$ be a signature of $W$. If $b_{1}, \cdots, b_{k} \in G$, then $\sigma\left(b_{i}\right)= \pm 1$ so $\sigma\left(1,-b_{1}\right) \otimes\left(1,-b_{2}\right) \otimes \cdots \otimes\left(1,-b_{k}\right)=0$ or $2^{k}$. Thus $\sigma\left(I^{k}\right) \cong 2^{k} Z$. On the other hand, clearly $|\sigma(f)| \leqq \operatorname{dim}(f)<2^{k}$. Thus $\sigma(f)=0$ for all signatures $\sigma$ of $W$. It follows, from 3.9 (ii), that $f$ is torsion. Thus, by assumption, $f=0$.

Recall $[5,2.24]$ that if $W$ is an abstract Witt ring which is representational, then so is the reduced ring $W_{\text {red }}=W / \mathrm{Nil}(W)$. Moreover (by [5, 2.30]), the abstract Witt rings which are reduced and representational are just the Witt rings of spaces of orderings in the terminology of [9]. It follows from 3.8 (ii) and 3.10 that all 
such rings are included in the theory presented here, i.e., are of the form $W(q)$ for some linked quaternionic mapping $q$. (By 3.9 (iii), $W$ is reduced if and only if $I$ is torsion free, so 3.10 applies.) Here is a characterization of the class of linked quaternionic mappings thus obtained.

TheOREM 3.11. Let $q: G \times G \rightarrow B$ be a linked quaternionic mapping. Then $W(q)$ is reduced if and only if $q$ satisfies

$$
q(a, a)=1 \Rightarrow a=1 \text {. }
$$

Proof. By 3.9 (i) and (iii), $W(q)$ is reduced if and only if

$$
2 \times f \sim 0 \Rightarrow f \sim 0 \quad \forall \text { even dimensional forms } f \text { over } G \text {. }
$$

Thus we must verify $(R) \Leftrightarrow\left(R^{\prime}\right)$. Assume $\left(R^{\prime}\right)$ and $q(a, a)=1$. Thus $(a, a) \cong(1,1)$, i.e., $2 \times(1,-a) \sim 0$. Thus, by $\left(R^{\prime}\right),(1,-a) \sim 0$, i.e., $a=1$. Thus $\left(R^{\prime}\right) \Rightarrow(R)$. Now assume $(R)$.

Claim. $D(2 \times f)=D(f) \forall$ forms $f$ over $G$. For suppose $f=$ $\left(a_{1}, \cdots, a_{n}\right)$, and that $x$ is represented by $2 \times f \cong\left(a_{1}, a_{1}\right) \oplus \cdots \oplus$ $\left(a_{n}, a_{n}\right)$. Thus, by a repeated application of 2.8., $\exists x_{i} \in D\left(a_{i}, a_{i}\right)$ such that $x \in D\left(x_{1}, \cdots, x_{n}\right)$. But $\left(a_{i}, a_{i}\right) \cong\left(x_{i}, x_{i}\right)$, i.e., $\left(a_{i} x_{i}, a_{i} x_{i}\right) \cong(1,1)$, i.e., $q\left(a_{i} x_{i}, a_{i} x_{i}\right)=1$, so by $(R), x_{i}=a_{i} \forall i=1, \cdots, n$. This proves $x \in D(f)$ and hence proves the claim.

Now suppose $\left(R^{\prime}\right)$ fails. Then $\exists$ an anisotropic form $f=$ $\left(a_{1}, \cdots, a_{n}\right)$ with $n$ even, $n \geqq 2$ and $2 \times f \sim 0$. But then $2 \times\left(a_{1}\right) \oplus$ $2 \times\left(a_{2}, \cdots, a_{n}\right) \sim 0$, so by 2.9 and the claim, $-a_{1} \in D\left(a_{2}, \cdots, a_{n}\right)$. This contradicts the fact that $f$ is anisotropic. Thus $(R) \Rightarrow\left(R^{\prime}\right)$.

\section{REFERENCES}

1. J.K. Arason and A. Pfister, Beweis des Krullschen Durchschnittsatzes für den Wittring, Inv. Math., 12 (1971), 173-176.

2. R. Baeza, Quadratic Forms over Semilocal Rings, Lecture notes in Math. 655, Springer-Verlag, 1978.

3. R. Baeza, M. Knebush, Annullatoren von Pfisterformen über semilokaten Ringen, Math. Z., 140 (1974), 41-62.

4. I. Kaplansky and R. Shaker, Abstract quadratic forms, Canad J. Math., 21 (1969), 1218-1233.

5. J.L. Kleinstein and A. Rosenberg, Succinct and representational Witt rings, Pacific J. Math., 86 (1980), 99-137.

6. M. Knebusch, Symmetric bilinear forms over algebraic varieties, Conference on quadratic forms 1976. (Ed. G. Orzech.) Queen's papers in pure and app. Math., 46 (1977).

7. M. Knebusch, A. Rosenberg and R. Ware, Structure of Witt rings and quotients of Abelian group rings, Amer. J. Math., 94 (1972), 119-155.

8. T. Y. Lam, The algebraic Theory of Quadratic Forms, Benjamin, 1973.

9. M. Marshall, The Witt ring of a space of orderings, to appear, AMS Trans. 
10. A. Pfister, Quadratische Formen in beliebigen Korpern, Inv. Math., 1 (1966), 116-132.

11. E. Witt, Theorie der quadratischen Formen in beliebigen Korpern, J. reine und ang. Math., 176 (1937), 31-44.

Received August 9, 1979 and in revised form April 14, 1980.

UNIVERSITY OF SASKATCHEWAN

SASKatoon, CaNADa S7N OWO

AND

The Pennsylvania State University

UnIVERSity PARK, PA 16802 



\section{PACIFIC JOURNAL OF MATHEMATICS}

\section{EDITORS}

DONALD BABBITT (Managing Editor)

University of California

Los Angeles, CA 90024

HUGo RossI

University of Utah

Salt Lake City, UT 84112

C. C. MOORE and ANDREW OGG

University of California

Berkeley, CA 94720
J. DugundjI

Department of Mathematics

University of Southern California

Los Angeles, CA 90007

R. FinN and J. Milgram

Stanford University

Stanford, CA 94305

\section{ASSOCIATE EDITORS}
R. ARENS
E. F. BECKENBACH
B. H. NeumanN
F. WOLF
K. YOSHIDA

\section{SUPPORTING INSTITUTIONS}

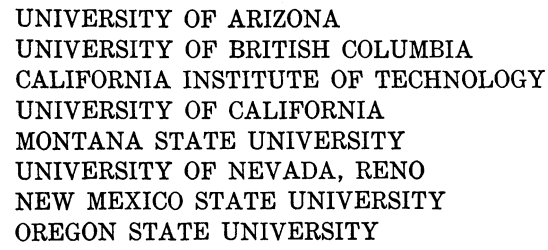

UNIVERSITY OF ARIZONA

UNIVERSITY OF BRITISH COLUMBIA

CALIFORNIA INSTITUTE OF TECHNOLOGY

UNIVERSITY OF CALIFORNIA

MONTANA STATE UNIVERSITY

UNIVERSITY OF NEVADA, RENO

NEW MEXICO STATE UNIVERSITY

OREGON STATE UNIVERSITY

\author{
UNIVERSITY OF OREGON \\ UNIVERSITY OF SOUTHERN CALIFORNIA \\ STANFORD UNIVERSITY \\ UNIVERSITY OF HAWAII \\ UNIVERSITY OF TOKYO \\ UNIVERSITY OF UTAH \\ WASHINGTON STATE UNIVERSITY \\ UNIVERSITY OF WASHINGTON
}

The Supporting Institutions listed above contribute to the cost of publication of this Journal, but they are not owners or publishers and have no responsibility for its content or policies.

Mathematical papers intended for publication in the Pacific Journal of Mathematics should be in typed form or offset-reproduced, (not dittoed), double spaced with large margins. Please do not use built up fractions in the text of the manuscript. However, you may use them in the displayed equations. Underline Greek letters in red, German in green, and script in blue. The first paragraph or two must be capable of being used separately as a synopsis of the entire paper. Please propose a heading for the odd numbered pages of less than 35 characters. Manuscripts, in triplicate, may be sent to any one of the editors. Please classify according to the scheme of Math. Reviews, Index to Vol. 39. Supply name and address of author to whom proofs should be sent. All other communications should be addressed to the managing editor, or Elaine Barth, University of California, Los Angeles, California, 90024.

50 reprints to each author are provided free for each article, only if page charges have been substantially paid. Additional copies may be obtained at cost in multiples of 50 .

The Pacific Journal of Mathematics is issued monthly as of January 1966. Regular subscription rate: $\$ 102.00$ a year (6 Vols., 12 issues). Special rate: $\$ 51.00$ a year to individual members of supporting institutions.

Subscriptions, orders for numbers issued in the last three calendar years, and changes of address shoud be sent to Pacific Journal of Mathematics, P.O. Box 969, Carmel Valley, CA 93924, U.S.A. Old back numbers obtainable from Kraus Per!odicals Co., Route 100, Millwood, NY 10546.

PUBLISHED BY PACIFIC JOURNAL OF MATHEMATICS, A NON-PROFIT CORPORATION

Printed at Kokusai Bunken Insatsusha (International Academic Printing Co., Ltd.). 8-8, 3-chome, Takadanobaba, Shinjuku-ku, Tokyo 160, Japan. 


\section{Pacific Journal of Mathematics}

\section{Vol. 95, No. $2 \quad$ October, 1981}

George E. Andrews, The Rogers-Ramanujan reciprocal and Minc's

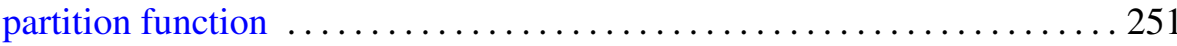

Allan Calder, William H. Julian, Ray Mines, III and Fred Richman,

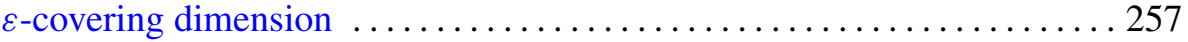

Thomas Curtis Craven and George Leslie Csordas, An inequality for the distribution of zeros of polynomials and entire functions $\ldots \ldots \ldots \ldots 263$

Thomas Jones Enright and R. Parthasarathy, The transfer of invariant

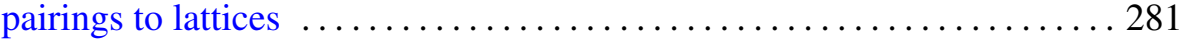

Allen Roy Freedman and John Joseph Sember, Densities and

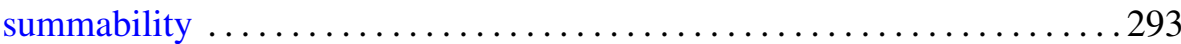

Robert Heller and Francis Aubra Roach, A generalization of a classical necessary condition for convergence of continued fractions . . . . . . 307

Peter Wilcox Jones, Ratios of interpolating Blaschke products ........... 311

V. J. Joseph, Smooth actions of the circle group on exotic spheres ........ 323

Mohd Saeed Khan, Common fixed point theorems for multivalued

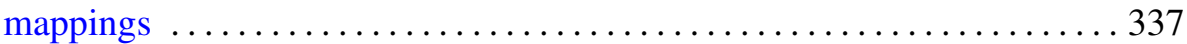

Samuel James Lomonaco, Jr., The homotopy groups of knots. I. How to

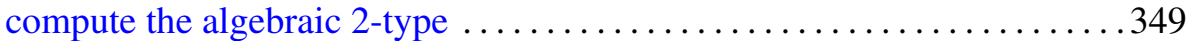

Louis Magnin, Some remarks about $C^{\infty}$ vectors in representations of connected locally compact groups ............................ 391

Mark Mandelker, Located sets on the line . . . . . . . . . . . . . . . . . 401

Murray Angus Marshall and Joseph Lewis Yucas, Linked quaternionic mappings and their associated Witt rings $\ldots \ldots \ldots \ldots \ldots \ldots \ldots \ldots . \ldots \ldots 11$

William Lindall Paschke, $K$-theory for commutants in the Calkin algebra

W. J. Phillips, On the relation $P Q-Q P=-i I$ 435

Ellen Elizabeth Reed, A class of Wallman-type extension. 443

Sungwoo Suh, The space of real parts of algebras of Fourier transforms 461 Antonius Johannes Van Haagen, Finite signed measures on function

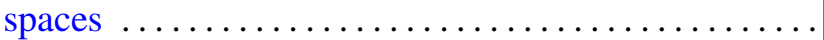

Richard Hawks Warren, Identification spaces and unique uniformity 